\title{
Catalpol preserves neural function and attenuates the pathology of Alzheimer's disease in mice
}

\author{
JIN-ZHONG HUANG ${ }^{1}$, JIAN WU $^{1}$, SHOUKUI XIANG ${ }^{2}$, SHIYING SHENG ${ }^{1}$, \\ YING JIANG $^{1}$, ZHILONG YANG ${ }^{1}$ and FEI HUA ${ }^{2}$ \\ Departments of ${ }^{1}$ Neurology and ${ }^{2}$ Endocrinology, The Third Affiliated Hospital of Soochow University, \\ Changzhou, Jiangsu 213000, P.R. China
}

Received January 3, 2015; Accepted October 2, 2015

DOI: $10.3892 / \mathrm{mmr} .2015 .4496$

\begin{abstract}
Alzheimer's disease (AD) is the most common form of dementia and there currently are no effective treatment strategies available. Catalpol is an iridoid glucoside, and large quantities can be isolated from the genus Rehmannia (Orobanchaceae). The present study assessed whether catalpol had any protective effects against Alzheimer's disease using a murine model. Reactive oxygen species (ROS)-associated enzymes as well as soluble $A \beta 40$ and $A \beta 42$ were detected using kits. Thioflavin-S staining was performed to detect senile plaques and reverse-transcription quantitative polymerase chain reaction was used to assess iroquois homeobox protein 3 (IRX3) and obesity-associated genes, while western blot analysis was used for $\beta$-secretase 1 (BACE1), insulin-degrading enzyme (IDE) and neprilysin (NEP) detection. The Morris water maze was used to detect the learning ability and spatial memory. The results revealed that catalpol was able to reduce the oxidative stress in the cerebral cortex by regulating the activities and concentration of ROS-associated enzymes superoxide dismutase, glutathione peroxidase and catalase, however not malondialdehyde. Catalpol was also identified to be able to reduce the levels of soluble $A \beta_{40}$ and $A \beta_{42}$ in the cerebral cortex and thus inhibit the formation of senile plaques. These effects were observed to be regulated by IDE, however not by BACE1 or NEP. It is suggested that catalpol is not capable of directly regulating the expression of IRX3 and obesity-associated genes. Subsequent to the treatment with catalpol, impairments in learning and memory were also observed to be relieved using the Morris water maze test. The results of the present study indicate that catalpol may be a
\end{abstract}

Correspondence to: Professor Fei Hua, Department of Endocrinology, The Third Affiliated Hospital of Soochow University, 185 Juqian Street, Changzhou, Jiangsu 213000, P.R. China

E-mail: feihua_changzhou@126.com

Key words: catalpol, Alzheimer's disease, neuroprotection, oxidative stress, $\beta$-amyloid, senile plaque, iroquois homeobox protein 3 , obesity potential drug for the treatment of neurodegenerative diseases such as AD.

\section{Introduction}

Alzheimer's disease (AD), the most common form of dementia, which currently has no effective available treatment, is commonly diagnosed in individuals over 65 years old (1). There are numerous common symptoms including confusion, irritability, aggression, mood swings, trouble with language and long-term memory loss, which ultimately leads to mortality. On average, the life expectancy following diagnosis is 3.2-6.6 years (2). $\beta$-amyloid (A $\beta$ ) can aggregate to form flexible soluble oligomers, which may exist in several forms. Senile plaques are extracellular deposits of $A \beta$ in the brain associated with degenerative neurons and an abundance of microglia and astrocytes (3). As a neurodegenerative disorder, $\mathrm{AD}$ is associated with the presence of these neurotoxic senile plaques in the brain (4). Oxidative stress is another important independent adverse factor in the development of AD (5).

Obesity may increase the risk of various diseases such as heart disease, type 2 diabetes, obstructive sleep apnea, certain types of cancer and osteoarthritis. Given that obesity is associated with vascular and metabolic diseases, it is suggested that it is associated with $\mathrm{AD}(6)$. As a novel determinant of body weight and obesity, iroquois homeobox protein 3 (IRX3) serves as a functional long-range target of obesity-associated variants within fat mass and obesity-associated (FTO) genes, influencing obesity (7). It is a member of the iroquois homeobox gene family and serves a role in the early stages of neural development (8). Obesity-associated single nucleotide polymorphisms have been demonstrated to be associated with IRX3 instead of fat mass and obesity-associated protein in the human brain (7).

Catalpol is an iridoid glucoside, and large quantities can be isolated from the genus Rehmannia (Orobanchaceae). Previous studies have indicated that catalpol serves a role in neuroprotection, however, the precise mechanisms remain unclear $(9,10)$. The current study predominantly focuses on elucidating whether catalpol preserves neural function and reduces the formation of senile plaques in $\mathrm{AD}$ using an $\mathrm{AD}$ mice model. In addition, the effects of catalpol on IRX3 and obesity-associated genes were investigated. 


\section{Materials and methods}

Generation of $A D$ mice. A total of 18 male 8-month-old Kunming mice provided by the Animal Center of The Third Affiliated Hospital of Soochow University (Changzhou, China). Mice were maintained under $50 \pm 5 \%$ relative humidity at $22 \pm 2^{\circ} \mathrm{C}$ with a 12 -h light/dark cycle and free access to food and water. The mice were randomly divided into 3 groups with 6 mice/group: The AD group; AD mice treated with catalpol group $(\mathrm{AD}+\mathrm{C})$; and the control group $(\mathrm{CON})$. For generation of the AD mouse model, 12 mice (6 each from the $\mathrm{AD}$ and $\mathrm{AD}+\mathrm{C}$ groups) were injected intraperitoneally with D-(+)-galactose (120 mg/kg; Sigma-Aldrich, St. Louis, MO, USA) and sodium nitrite $(90 \mathrm{mg} / \mathrm{kg}$; Sigma-Aldrich), once daily continuously for 60 days. In order to enhance the progression of senile dementia, a one-off injection of $2 \mu \mathrm{l} \mathrm{A} \beta_{1-42}$ (amino acid sequence: DAEFGHDSGFEVRHQ KLVFFAEDVGSNKGAIIGLMVGGVVIA; synthesized by Sangon Biotech Co., Ltd., Shanghai, China) into the left lateral ventricle of the 12 mice was conducted when the mice were 9-months old. At 10 months, the mice were considered to have AD. Catalpol ( $20 \mathrm{mg} / \mathrm{kg}$; Sigma-Aldrich) was administered at 10 months for 30 days by intragastric administration in the $\mathrm{AD}+\mathrm{C}$ group. No further treatment was administered in the $\mathrm{AD}$ group. The $\mathrm{CON}$ group mice were 11-months old without any chemical treatment. All mice used in the experiments of the current study were 11 months old. All experiments were approved by the Ethics Committee of Soochow University (Suzhou, China).

Detection of reactive oxygen species (ROS)-associated enzymes. Following sacrification of the mice by $\mathrm{CO}_{2}$ inhalation, the cerebral cortex of the mouse brain was dissected and centrifuged at a speed of $12,000 \mathrm{x} \mathrm{g}$ for $15 \mathrm{~min}$ in Tris-buffered saline with Protease Inhibitor Cocktail (1:1,000; Sigma-Aldrich) for $30 \mathrm{~min}$ at $4^{\circ} \mathrm{C}$. The supernatant of the cerebral cortex was extracted and transferred to a tube. ROS-associated enzymes from homogenates were detected using the following kits purchased from Beyotime Institute of Biotechnology (Haimen, China): Superoxide dismutase (SOD), glutathione peroxidase (GSH-Px3), catalase (CAT) and malondialdehyde (MDH).

Detection of soluble $A \beta_{40}$ and $A \beta_{42}$. The above-mentioned supernatant containing soluble $A \beta$ was homogenized in $5 \mathrm{M}$ guanidine $\mathrm{HCl}$ and $50 \mathrm{mM}$ Tris- $\mathrm{HCl}(\mathrm{pH}$ 8.0) buffer, and aliquots of the homogenates were diluted 40 fold. The supernatant was used for the determination of the concentrations of $A \beta_{40}$ and $A \beta_{42}$ peptides using Mouse ELISA kits (Thermo Fisher Scientific, Waltham, MA, USA). The optical density value was detected at $\lambda=495 \mathrm{~nm}$ using the PR-310 ELISA reader (Shenzhen Procan Electronics Inc., Shenzhen, China).

Thioflavin-S staining. Mouse brains in the three groups were dissected and embedded in optimum cutting temperature compound (Sakura Finetek USA Inc., Torrance, CA, USA) and rapidly frozen on dry ice. Coronal sections $(10 \mu \mathrm{m})$ were cut and deparaffinized in $4 \%$ paraformaldehyde for $20 \mathrm{~min}$. Slides were placed in 1\% thioflavin-S solution (catalog no. T1892; Sigma-Aldrich) for $12 \mathrm{~min}$, then were differentiated twice in $70 \%$ fresh alcohol for $10 \mathrm{~min}$. Subsequent to rinsing in distilled water, the slides were mounted in glycerin jelly. Images were captured using a confocal microscope (A1R+; Nikon Corporation, Tokyo, Japan) and analyzed by the relative area (\%) of senile plaques (white spot as indicated by the arrows) using ImageJ software (National Institutes of Health, Bethesda, MD, USA).

Detection of IRX3 and obesity-associated genes. The expression of obesity-associated genes and IRX3 at the gene level was conducted using reverse transcription-quantitative polymerase chain reaction (RT-qPCR) using the ABI 7500 cycler (Applied Biosystems; Thermo Fisher Scientific). Total RNA was isolated from three groups using TRIzol ${ }^{\circledR}$ reagent (Ambion; Thermo Fisher Scientific) according to the manufacturer's instructions. For each group, $2.5 \mu \mathrm{g}$ RNA in a $20-\mu 1$ reaction mixture was reverse-transcribed into cDNA using the SuperScript ${ }^{\circledR}$ VILO $^{\mathrm{TM}}$ cDNA Synthesis kit (Invitrogen; Thermo Fisher Scientific) according to the manufacturer's instructions. Briefly, tube contents were mixed and incubated at $25^{\circ} \mathrm{C}$ for $10 \mathrm{~min}$, then incubated at $42^{\circ} \mathrm{C}$ for $1 \mathrm{~h}$ and terminated at $85^{\circ} \mathrm{C}$ for $5 \mathrm{~min}$. The products of cDNA were used as templates in the following qPCR. The following cycling conditions were used: Stage 1,1 cycle of $95^{\circ} \mathrm{C}$ for $30 \mathrm{sec}$; stage 2,40 cycles of $95^{\circ} \mathrm{C}$ for $5 \mathrm{sec}$ and $60^{\circ} \mathrm{C}$ for $20 \mathrm{sec}$; and stage 3,1 cycle of $65^{\circ} \mathrm{C}$ for $15 \mathrm{sec}$. The Obesity PCR Array was performed using a 96-well plate (SABiosciences; Qiagen Inc., Valencia, CA, USA) to detect the obesity-associated genes, which predominantly include orexigenic genes, anorectic genes and genes associated with energy expenditure. The PCR array was a set of optimized primer assays in a 96-well plate and was used for gene expression analysis. cDNA template was mixed with the PCR master mix, and then run the PCR program The 102 bp primer sequence for IRX3 (Sangon Biotech Co., Ltd.) was as follows: Forward, 5'-GAAAACTTAGACAGC GCGGCAG-3' and reverse, 5'-AGTTTTGCAGTCCGAAA TGGGT-3'. cDNA in these groups was reverse transcribed and used as templates with the SYBR ${ }^{\circledR}$ Premix Ex Taq ${ }^{\mathrm{TM}}$ II (Perfect Real Time) kit (Takara Biotechnology Co., Ltd., Dalian, China). Gene expression values were determined relative to that of the control group, which was set as $1.2^{-\Delta \Delta \mathrm{CT}}$ was used for analysis (11)

Western blot analysis. A total of $30 \mu \mathrm{g}$ protein isolated from the mouse cortex was boiled for $5 \mathrm{~min}$ then cooled on ice and injected into 10\% SDS-PAGE for electrophoresis. The proteins were then transferred onto a Immobilon-PFL polyvinylidene fluoride membrane (Millipore, Billerica, MA, USA) and blocked in 5\% fat-free milk for $1 \mathrm{~h}$. Membranes were incubated overnight at $4^{\circ} \mathrm{C}$ with rabbit polyclonal immunoglobulin (Ig)G anti-BACE1 (70 kDa; 1:1,000; cat. no. ab2077; Abcam, Cambridge,UK), rabbit polyclonal IgG anti-IDE (118 kDa; 1:1,000; catalog no. ab32216; Abcam), rabbit polyclonal IgG anti-NEP (86 kDa; catalog no. AB5458; Chemicon; EMD Millipore) and rabbit monoclonal IgG anti-IRX3 (52 kDa; 1:1,500; cat. no. ab174307; Abcam) primary antibodies, and for $1 \mathrm{~h}$ at room temperature followed by three washes with phosphate-buffered saline for $10 \mathrm{~min}$ each. Subsequently, the membranes were incubated with the alkaline phosphatase-conjugated goat anti-rabbit IgG secondary antibody (1:2,000; cat. no. 65-6122; Thermo Fisher 
A

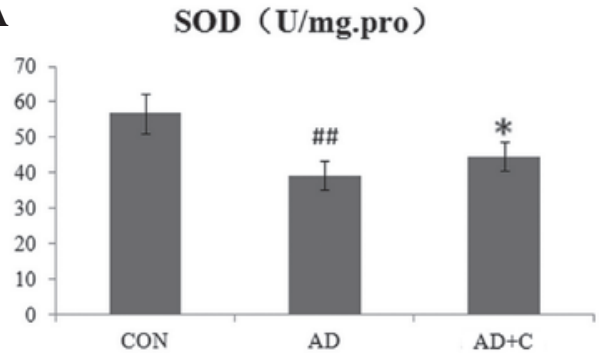

C

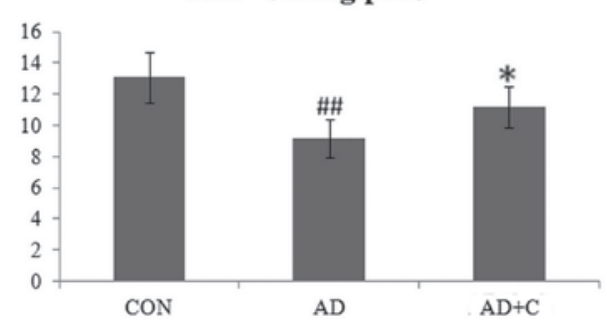

B

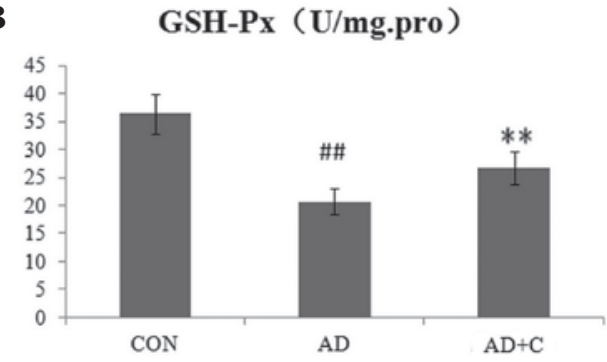

D

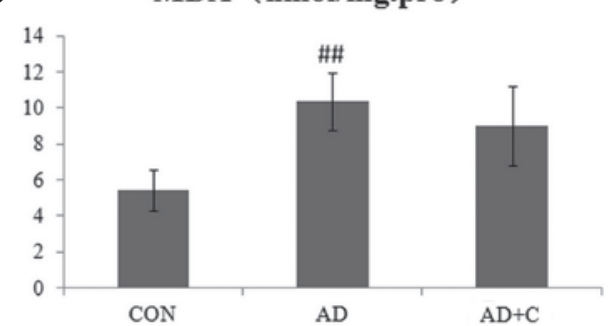

Figure 1. Detection of reactive oxygen species-associated enzymes in the supernatant of the cerebral cortex of mouse brains in 3 groups. (A) SOD; (B) GSH-Px; (C) CAT; and (D) MDA. Data are presented as the mean \pm standard deviation. ${ }^{\# \#} \mathrm{P}<0.01$, vs. $\mathrm{CON}$; ${ }^{*} \mathrm{P}<0.05$, vs. AD; ${ }^{* *} \mathrm{P}<0.01$, vs. AD. SOD, superoxide dismutase; GSH-Px, glutathione peroxidase; CAT, catalase; MDA, malondialdehyde; AD, Alzheimer's disease; CON, control; C, catalpol.

Scientific). A polyclonal rabbit anti-glyceraldehyde 3-phosphate dehydrogenase antibody (cat. no. G9545; Sigma-Aldrich) was used as an internal control. Immunoreactive bands were visualized using Pierce ${ }^{\mathrm{TM}}$ ECL Substrate (Thermo Fisher Scientific) and then scanned (Fujifilm LAS 4000; Fujifilm, Tokyo, Japan).

Morris water maze test. The Morris water maze was used to detect the abilities of learning and memory of the spatial location via training mice to find a hidden platform. The swimming pool $(120 \mathrm{~cm}$ in diameter, $50 \mathrm{~cm}$ in height, $30 \mathrm{~cm}$ depth of water made opaque with milk) was divided into 4 equal quadrants. A submerged platform $(10 \mathrm{~cm}$ in diameter, $29 \mathrm{~cm}$ in height) was placed inside the target quadrant and $1 \mathrm{~cm}$ below the surface of the water while other three quadrants were entry points of the mice. Mice were trained to swim and find the hidden platform during the Morris water maze test for five consecutive days, including four days of place navigation with three consecutive trials per day, and the probe trial performed on the last day. On the testing day (day 5), the platform was removed and mice were allowed to explore the pool for $90 \mathrm{sec}$. The time (sec) spent in the target quadrant and the number of crossings of the former location of the hidden platform were recorded and considered as an index of spatial learning and memory.

Statistical analysis. Data are presented as the mean \pm standard deviation. Calculations were performed using SPSS software, version 16.0 (SPSS, Inc., Chicago, IL, USA). The results were evaluated by one-way analysis of variance. $\mathrm{P}<0.05$ was considered to indicate a statistically significant difference.

\section{Results}

Effects of catalpol on ROS-associated enzymes. The activities of the ROS-associated enzymes, SOD, GSH-Px and CAT, and the concentration of MDA were detected separately using the kits mentioned above. As shown in Fig. 1, the activity of SOD, GSH-Px and CAT was significantly reduced in the AD mice $(\mathrm{P}<0.01)$, which suggested the suppressive capacities of ROS clearance in the AD group. Subsequent to treatment with catalpol, the activity of these enzymes was increased significantly although were still lower than those of the CON group. The concentration of MDA was increased significantly in the AD group, suggesting the enhanced induction of ROS, however, no clear effect on MDA levels was detected following treatment with catalpol.

Effects of catalpol on reduction of $A \beta_{40}$ and $A \beta_{42}$. As presented in Fig. 2, the concentration of $A \beta_{40}$ (Fig. 2A) and $A \beta_{42}$ (Fig. 2B) in the cortex of the brain was increased in AD mice as expected. Subsequent to treatment with catalpol, the level of $A \beta_{40}$ and $A \beta_{42}$, particularly $A \beta_{40}$, in the $A D+C$ group reduced significantly but remained higher than the level in the $\mathrm{CON}$ group.

Effects of catalpol on the formation of senile plaques. Senile plaques in the hippocampus and cortex of mice were detected using thioflavin-S staining. As presented in Fig. 3A, the senile plaques (white spots indicated by arrows) were seldom detected in the hippocampus and cortex of mice in the CON group. Senile plaques were observed to be significantly increased in the AD group (Fig. 3B). Subsequent to treatment with catalpol, the number of senile plaques in the hippocampus and cortex were observed to be significantly reduced in the $\mathrm{AD}+\mathrm{C}$ group (Fig. 3C), but remained higher than that in the CON group. The result indicated that catalpol reduced the formation of senile plaques in the hippocampus and cortex. The quantification of the plaque area is presented in Fig. 3D.

Effects of catalpol on the expression levels of IRX3 and obesity-associated genes. As presented in Fig. 4A, no significant alterations in the expression levels of IRX 3 were detected at the gene level. Additionally, no significant alterations in the 

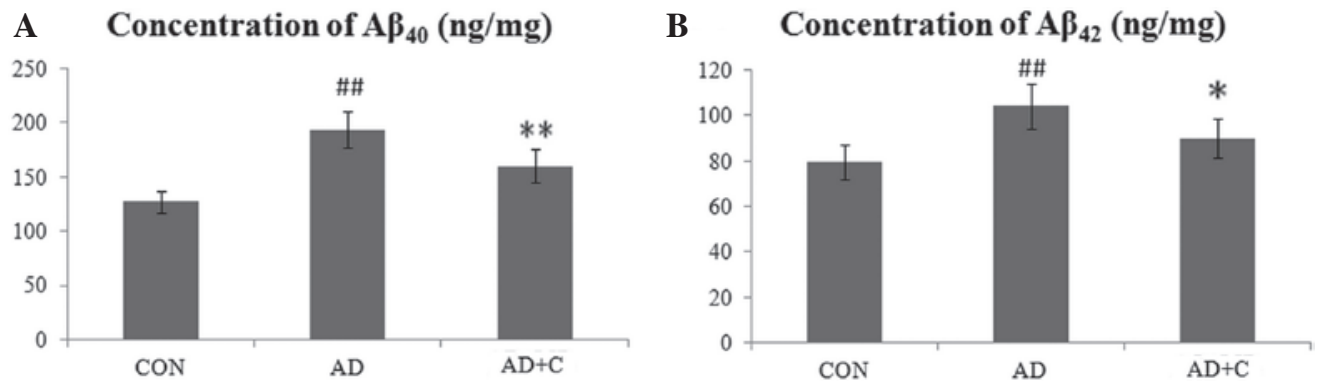

Figure 2. Concentrations of soluble $A \beta_{40}$ and $A \beta_{42}$ in the cerebral cortex of mouse brains in the 3 groups. (A) $A \beta_{40}$; and (B) $A \beta_{42}$. Data are presented as the mean \pm standard deviation. ${ }^{\# \#} \mathrm{P}<0.01$, vs. CON; ${ }^{\mathrm{P}}<0.05$, vs. AD; ${ }^{* *} \mathrm{P}<0.01$, vs. AD. A $\beta, \beta$-amyloid; AD, Alzheimer's disease; CON, control; $\mathrm{C}$, catalpol.
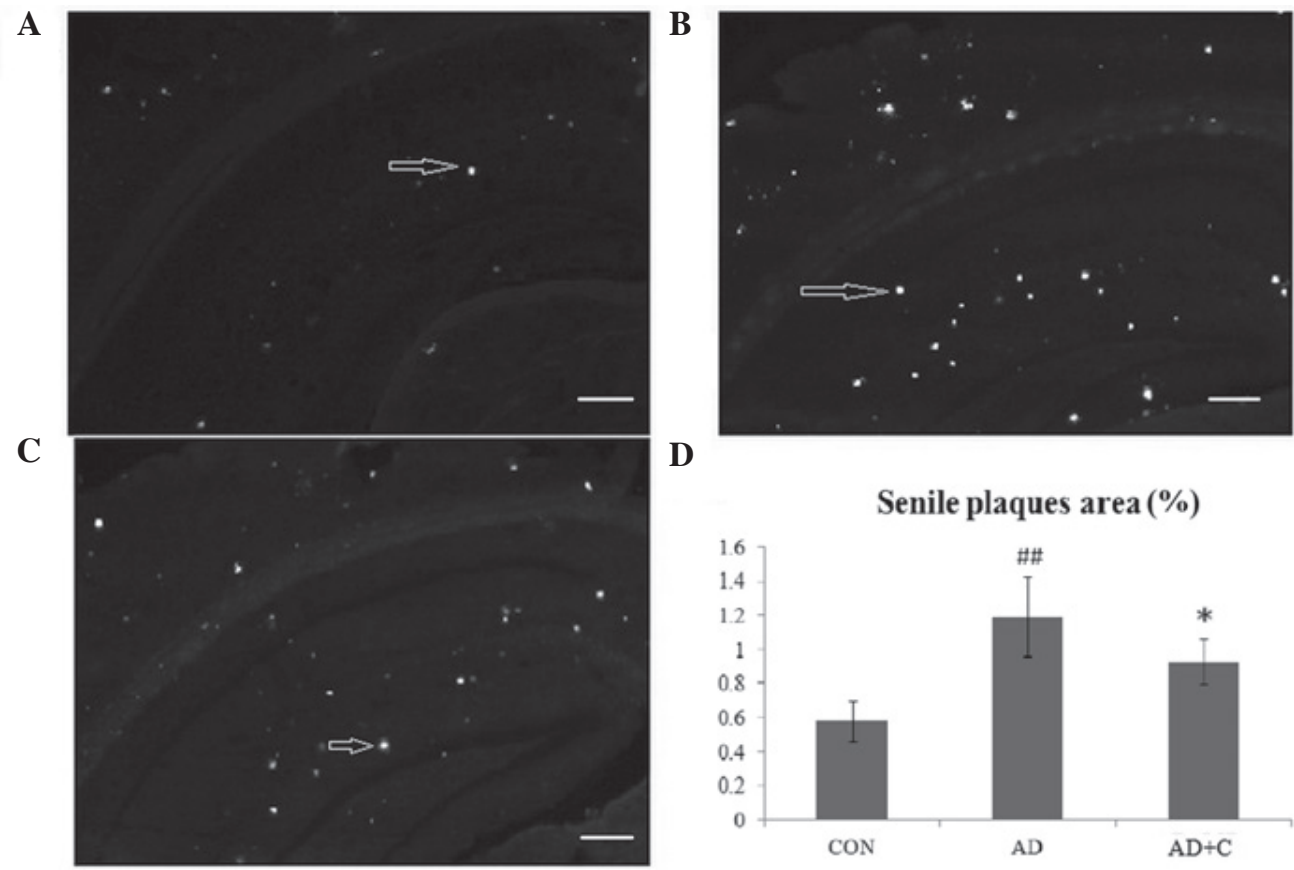

D

\section{Senile plaques area $(\%)$}

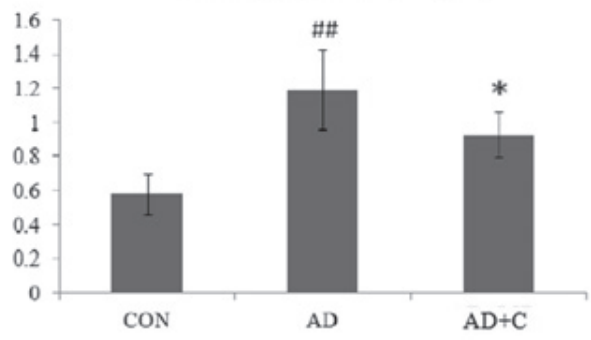

Figure 3. Senile plaques (white spots indicated by arrows; scale bar $=100 \mu \mathrm{m}$ ) in the hippocampus and cortex of mouse brains in the 3 groups. (A) CON group; (B) AD group; (C) AD+C group. (D) Relative area of senile plaques (\% of total area). Data are presented as the mean \pm standard deviation. ${ }^{\# \#} \mathrm{P}<0.01$, vs. CON; ${ }^{*} \mathrm{P}<0.05$, vs. AD. AD, Alzheimer's disease; CON, control; C, catalpol.

expression levels of obesity-associated genes were observed. In addition, no alterations in the protein expression levels of IRX3 were observed (Fig. 4B). These results indicated that catalpol was not able to regulate the levels of IRX3. The alterations were considered significant if the fold change was $>2$ fold (increased) or $<0.5$ fold (reduced).

Effects of catalpol on enzyme regulation. BACE-1, IDE and NEP are three key enzymes regulating the formation of senile plaques during the progression of $\mathrm{AD}$ (12). As presented in Fig. 4B, compared with the CON group, the expression of IDE was downregulated in the AD group. Subsequent to treatment with catalpol, the expression of IDE was observed to be significantly upregulated in the $\mathrm{AD}+\mathrm{C}$ group. However, no alterations to the expression of BACE-1 and NEP were detected among these groups. These results indicated that catalpol protected the brain by upregulating the expression of IDE.

Effects of catalpol on learning and memory promotion. The target quadrant time and the average number of hidden platform crossings in the $\mathrm{CON}, \mathrm{AD}$ and $\mathrm{AD}+\mathrm{C}$ groups were recorded. As presented in Fig. 5, the time spent in the target quadrant determined by the representative swim paths in the pool was longer in the CON group, however was significantly reduced in the AD group $(\mathrm{P}<0.01)$. The number of hidden platform crossings in the AD mice was significantly reduced when compared with that of the CON group $(\mathrm{P}<0.01)$. Following catalpol treatment, these data were increased compared with the AD group $(\mathrm{P}<0.05)$, but remained lower than that in the $\mathrm{CON}$ group. These results indicate the effects on learning and spatial memory in the AD mice, which may be ameliorated and by catalpol.

\section{Discussion}

Catalpol is an iridoid glucoside isolated from the genus Rehmannia (Orobanchaceae). Previous experiments by our group suggested that catalpol can stimulate adrenocortical hormones, increasing the production of androgens (unpublished data). Catalpol has additionally been identified to produce anti-inflammatory $(13,14)$, anticancer $(15,16)$, diuretic and 

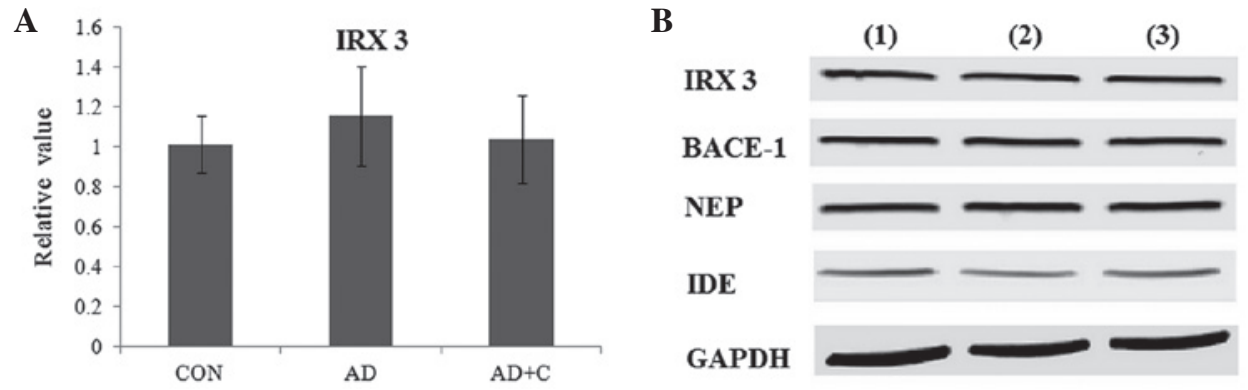

Figure 4. (A) The expression of IRX3 at the gene level in the mouse brain in 3 groups. Data are presented as the mean \pm standard deviation. (B) The expression of IRX3, BACE-1, IDE and NEP at the protein level in the mouse brains in 3 groups as observed by western blotting. GAPDH was used as an internal control. Lane 1, CON group; lane 2, AD group; lane 3, AD+C group. IRX3, iroquois homeobox protein 3; BACE-1, $\beta$-secretase 1; NEP, neprilysin; IDE, insulin-degrading enzyme; GAPDH, glyceraldehyde 3-phosphate dehydrogenase; CON, control; AD, Alzheimer's disease; C, catalpol.
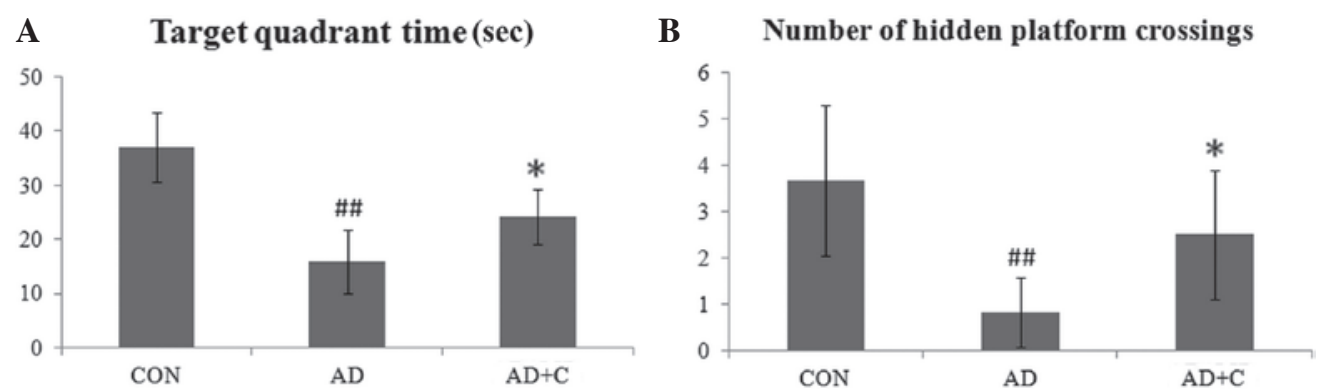

Figure 5. Morris water maze test in the 3 groups of mice. (A) The target quadrant time. (B) Number of hidden platform crossings. Data are presented as the mean \pm standard deviation. ${ }^{\# \#} \mathrm{P}<0.01$, vs. CON; "P<0.05, vs. AD. AD, Alzheimer's disease; CON, control; C, catalpol.

hypoglycemic effects $(17,18)$. Previously, catalpol was reported to exhibit neuroprotective effects, including protection against neurodegeneration (19). However, the mechanisms remain unclear.

Oxidative stress serves a key role in $\mathrm{AD}$, which is the most common form of dementia and currently has no cure. It reflects an imbalance in ROS, which is regulated by numerous enzymes, and damages the neurons in the brain. ROS are regulated by many enzymes. The activity of ROS-associated enzymes, SOD, GSH-Px and CAT, and the concentration of MDA are important in the process of $\mathrm{AD}$ (20). SOD catalyzes the dismutation of the toxic superoxide $\left(\mathrm{O}_{2}^{-}\right)$radical into $\mathrm{O}_{2}$ or $\mathrm{H}_{2} \mathrm{O}_{2}$. It is an important antioxidant in the majority of living cells exposed to oxygen. GSH-Px reduces lipid hydroperoxides to their corresponding alcohols and reduces free hydrogen peroxide to $\mathrm{H}_{2} \mathrm{O}$. CAT catalyzes the decomposition of hydrogen peroxide to water and oxygen, and is an important enzyme in protecting the cell from oxidative damage. MDA is a marker of oxidative stress as ROS degrades polyunsaturated lipids, forming MDA. The activities of these enzymes were observed to be significantly reduced in AD mice following the progression of disease. Subsequent to treatment with catalpol, the activities of these enzymes were increased significantly but remained lower than those of the CON group. These results suggested that catalpol may partially ameliorate the effects on the levels of these enzymes. Notably, there was no clear inhibition on MDA detected following treatment with catalpol, however this requires further investigation in order to be verified.

Previous studies suggest that $A \beta$, which is a peptide of $36-43$ amino acids in length, serves a central role in the pathological development of $\mathrm{AD}$; increases in $\mathrm{A} \beta_{40}$ and $\mathrm{A} \beta_{42}$ have been implicated in the pathogenesis of $\mathrm{AD}(21,22)$. Levels of $\mathrm{A} \beta_{40}$ and $A \beta_{42}$, which aggregate to form flexible soluble oligomers that are toxic to neurons, were observed to be significantly elevated in the brains of patients with AD (23). Senile plaques, the presence of which are an important criterion of the neurohistopathological verification of $A D$, contain $A \beta_{40}$ and $A \beta_{42}$ (24). In the current study, the concentrations of $A \beta_{40}$ and $\mathrm{A} \beta_{42}$ in the cortex of the brain were increased in AD mice as hypothesized. Following treatment with catalpol, the levels of $A \beta_{40}$ and $A \beta_{42}$, particularly $A \beta_{40}$, in the $A D+C$ group reduced significantly but remained higher than the levels in the CON group. Thus, catalpol was shown to reduce the formation of senile plaques in the hippocampus and cortex, which are increased in AD. A $\beta$ is removed and cleaved by several amyloid-degrading enzymes, including BACE-1, IDE and NEP (12). The results of the current study indicated that catalpol protected the brain by upregulating IDE, rather than the other two enzymes.

IRX3, a member of the iroquois homeobox gene family, serves a key role in early stages of neural development. A previous study demonstrated tha IRX3 is involved in neural formation and growth. IRX3 was previously identified to influence obesity (7). However, no alterations on the expression of IRX3 were detected at the gene and protein levels, thus suggesting that catalpol was not able to regulate IRX3. In addition, no significant alterations in the expression of obesity-associated genes were observed following treatment with catalpol. Although obesity has an important role in AD due to vascular defects, impaired insulin metabolism and defects in glucose transport in the brain, the results indicated that catalpol is not capable of regulating obesity. 
The Morris water maze test is widely used to investigate spatial learning and memory, whilst it has been additionally used to measure the effect of drugs on neurocognitive disorders (25). The current study identified that catalpol was able to increase the time spent in the target quadrant and the average number of hidden platform crossings, suggesting that the effects on learning and spatial memory in the AD mice were partially ameliorated by catalpol.

In conclusion, the present study indicates that catalpol reduces the levels of oxidative stress in the cerebral cortex of the hippocampus by regulating SOD, GSH-Px and CAT. Catalpol is additionally suggested to reduce the levels of soluble $A \beta_{40}$ and $\mathrm{A} \beta_{42}$ in the cerebral cortex of the hippocampus and thus inhibit the formation of senile plaques, which are regulated by IDE. Catalpol is not able to directly regulate the expression of IRX3 and obesity-associated genes. The learning and memory impairments were additionally identified to be relieved following treatment with catalpol. Thus, catalpol may be a potential drug for the treatment of neurodegenerative diseases such as AD.

\section{Acknowledgements}

The current study was supported by the Third Affiliated Hospital of Soochow University.

\section{References}

1. Forsyth E and Ritzline PD: An overview of the etiology, diagnosis, and treatment of Alzheimer disease. Phys Ther 78: 1325-1331, 1998.

2. Todd S, Barr S, Roberts M and Passmore AP: Survival in dementia and predictors of mortality: A review. Int J Geriatr Psychiatry 28 : 1109-1124, 2013.

3. Jang BG, In S, Choi B and Kim MJ: Beta-amyloid oligomers induce early loss of presynaptic proteins in primary neurons by caspase-dependent and proteasome-dependent mechanisms. Neuroreport 25: 1281-1288, 2014.

4. Henriques AG, Oliveira JM, Gomes B, Ruivo R, da Cruz e Silva EF and da Cruz e Silva OA: Complexing A $\beta$ prevents the cellular anomalies induced by the Peptide alone. J Mol Neurosci 53 661-668, 2014.

5. Shichiri M: The role of lipid peroxidation in neurological disorders. J Clin Biochem Nutr 54: 151-160, 2014.

6. Spielman LJ, Little JP and Klegeris A: Inflammation and insulin/IGF-1 resistance as the possible link between obesity and neurodegeneration. J Neuroimmunol 273: 8-21, 2014.

7. Smemo S, Tena JJ, Kim KH, Gamazon ER, Sakabe NJ, Gómez-Marín C, Aneas I, Credidio FL, Sobreira DR Wasserman NF, et al: Obesity-associated variants within FTO form long-range functional connections with IRX3. Nature 507: $371-375,2014$

8. Rodríguez-Seguel E, Alarcón P and Gómez-Skarmeta JL: The Xenopus Irx genes are essential for neural patterning and define the border between prethalamus and thalamus through mutual antagonism with the anterior repressors Fezf and Arx. Dev Biol 329: 258-268, 2009
9. Cai QY, Chen XS, Zhan XL and Yao ZX: Protective effects of catalpol on oligodendrocyte death and myelin breakdown in a rat model of chronic cerebral hypoperfusion. Neurosci Lett 497: 22-26, 2011

10. Li DQ, Li Y, Liu Y, Bao YM, Hu B and An LJ: Catalpol prevents the loss of CA1 hippocampal neurons and reduces working errors in gerbils after ischemia-reperfusion injury. Toxicon 46: 845-851, 2005.

11. Miners JS, van Helmond Z, Kehoe PG and Love S: Changes with age in the activities of beta-secretase and the Abeta-degrading enzymes neprilysin, insulin-degrading enzyme and angiotensin-converting enzyme. Brain Pathol 20: 794-802, 2010.

12. Choi HJ, Jang HJ, Chung TW, Jeong SI, Cha J, Choi JY, Han CW, Jang YS, Joo M, Jeong HS and Ha KT: Catalpol suppresses advanced glycation end-products-induced inflammatory responses through inhibition of reactive oxygen species in human monocytic THP-1 cells. Fitoterapia 86: 19-28, 2013.

13. Zhang A, Hao S, Bi J, Bao Y, Zhang X, An L and Jiang B: Effects of catalpol on mitochondrial function and working memory in mice after lipopolysaccharide-induced acute systemic inflammation. Exp Toxicol Pathol 61: 461-469, 2009.

14. Gao N, Tian JX, Shang YH, Zhao DY and Wu T: Catalpol suppresses proliferation and facilitates apoptosis of OVCAR-3 ovarian cancer cells through upregulating microRNA-200 and downregulating MMP-2 expression. Int J Mol Sci 15: 19394-19405, 2014.

15. Pungitore CR, Ayub MJ, Borkowski EJ, Tonn CE and Ciuffo GM: Inhibition of Taq DNA polymerase by catalpol. Cell Mol Biol (Noisy-le-grand) 50: 767-772, 2004.

16. Li X, Xu Z, Jiang Z, Sun L, Ji J, Miao J, Zhang X, Huang S, Wang $T$ and Zhang L: Hypoglycemic effect of catalpol on high-fat diet/streptozotocin-induced diabetic mice by increasing skeletal muscle mitochondrial biogenesis. Acta Biochim Biophys Sin (Shanghai) 46: 738-748, 2014.

17. Huang WJ, Niu HS, Lin MH, Cheng JT and Hsu FL: Antihyperglycemic effect of catalpol in streptozotocin-induced diabetic rats. J Nat Prod 73: 1170-1172, 2010.

18. Xu G, Xiong Z, Yong Y, Wang Z, Ke Z, Xia Z and $\mathrm{Hu}$ Y: Catalpol attenuates MPTP induced neuronal degeneration of nigral-striatal dopaminergic pathway in mice through elevating glial cell derived neurotrophic factor in striatum. Neuroscience 167: 174-184, 2010.

19. Watson JB, Arnold MM, Ho YS and O'Dell TJ: Age-dependent modulation of hippocampal long-term potentiation by antioxidant enzymes. J Neurosci Res 84: 1564-1574, 2006.

20. Bondareff W: Age-related changes in brain extracellular space affect processing of amyloid- $\beta$ peptides in Alzheimer's disease. J Alzheimers Dis 35: 1-6, 2013.

21. Hampel H, Shen Y, Walsh DM, Aisen P, Shaw LM, Zetterberg H, Trojanowski JQ and Blennow K: Biological markers of amyloid beta-related mechanisms in Alzheimer's disease. Exp Neurol 223: 334-346, 2010.

22. Hashimoto M, Bogdanovic N, Volkmann I, Aoki M, Winblad B and Tjernberg LO: Analysis of microdissected human neurons by a sensitive ELISA reveals a correlation between elevated intracellular concentrations of Abeta42 and Alzheimer's disease neuropathology. Acta Neuropathol 119: 543-554, 2010.

23. Tian J, Shi J and Mann DM: Cerebral amyloid angiopathy and dementia. Panminerva Med 46: 253-264, 2004.

24. Bromley-Brits K, Deng Y and Song W: Morris water maze test for learning and memory deficits in Alzheimer's disease model mice. J Vis Exp 53: pii2920, 2011.

25. Livak KJ and Schmittgen TD: Analysis of relative gene expression data using real-time quantitative PCR and the 2(-Delta Delta C(T)) Method. Methods 25: 402-408, 2001 